\title{
Chimeric cellobiohydrolase I expression, activity, and biochemical properties in three oleaginous yeast
}

\author{
Markus Alahuhta* (1D, Qi Xu, Eric P. Knoshaug, Wei Wang, Hui Wei, Antonella Amore, John O. Baker, \\ Todd Vander Wall, Michael E. Himmel and Min Zhang*
}

\begin{abstract}
Consolidated bioprocessing using oleaginous yeast is a promising modality for the economic conversion of plant biomass to fuels and chemicals. However, yeast are not known to produce effective biomass degrading enzymes naturally and this trait is essential for efficient consolidated bioprocessing. We expressed a chimeric cellobiohydrolase I gene in three different oleaginous, industrially relevant yeast: Yarrowia lipolytica, Lipomyces starkeyi, and Saccharomyces cerevisiae to study the biochemical and catalytic properties and biomass deconstruction potential of these recombinant enzymes. Our results showed differences in glycosylation, surface charge, thermal and proteolytic stability, and efficacy of biomass digestion. L. starkeyi was shown to be an inferior active cellulase producer compared to both the Y. lipolytica and S. cerevisiae enzymes, whereas the cellulase expressed in S. cerevisiae displayed the lowest activity against dilute-acid-pretreated corn stover. Comparatively, the chimeric cellobiohydrolase I enzyme expressed in Y. lipolytica was found to have a lower extent of glycosylation, better protease stability, and higher activity against dilute-acid-pretreated corn stover.
\end{abstract}

Keywords: Metabolic engineering: chimeric protein, Oleaginous yeast, $\mathrm{CBH}$ I, Consolidated bioprocessing, Cellobiohydrolase, Cel7A

\section{Background}

Efficient deconstruction of lignocellulosic biomass for the purposes of the production of advanced biofuels is challenging. The natural recalcitrance of plant cell walls to degradation has led to several industrially relevant strategies to more efficiently produce monomeric, fermentable sugars from lignocellulosic biomass [1-4]. Fungal cellulase systems produce an array of non-peptide tethered glucoside hydrolases that act in concert to efficiently degrade lignocellulosic biomass $[5,6]$. Consolidated bioprocessing (CBP) uses microorganisms that can break down the cellulosic and hemicellulosic fractions

*Correspondence: markus.alahuhta@nrel.gov; min.zhang@nrel.gov Biosciences Center, National Renewable Energy Laboratory, Golden, CO 80401, USA of pretreated biomass and simultaneously convert the released monomeric sugars to useful products [7-9]. Yeast can produce high yields of biofuels or their molecular precursors, making them a particularly interesting choice for CBP. For example, ethanol from Saccharomyces cerevisiae [10] and fatty acids or lipids from Yarrowia lipolytica [11, 12], Lipomyces starkeyi [13-15], and S. cerevisiae [16, 17]. However, these yeast are not naturally cellulolytic and must be engineered to secrete cellulase enzymes [1,7].

To enable CBP capabilities in yeast, significant secretion levels of cellulases, particularly cellobiohydrolase I (CBH I) are needed [1, 18]. S. cerevisiae [7, 19-21], Y. lipolytica [22-24], L. starkeyi [25], or all three hosts [26] are often studied examples of yeast CBP organisms. Still, low secretion levels and yields of active enzymes have affected their usability [27-29]. To remedy this, different 
strategies have been attempted [30, 31]. For example, $\mathrm{CBH}$ I enzymes from different fungal species, including Neurospora crassa, are known to have performance properties similar to CBH I enzymes from Trichoderma reesei and have been screened and compared [22, 23]. Recently, $Y$. lipolytica has been engineered to degrade biomass allowing growth on Avicel [22, 23]. Furthermore, cell-surface expression of cellulases has shown promise $[32,33]$, and a tunable $S$. cerevisiae expression system has been shown to improve cellulolytic activity compared to a strain with three cellulase-expression constructs in a high-performance gene expression cassette [34].

Another strategy employed for fungal cellulase expression is making chimeric enzymes from domains of different fungal species that may be more easily secreted, such as the Talaromyces emersonii catalytic domain connected to the $T$. reesei linker peptide and carbohydrate binding module (CBM1) forming a chimeric $T e T r C B H ~ I$ [24]. Similarly, a fusion protein constructed with an easily secreted leading enzyme, endoglucanase II from $T$. reesei, followed by the more difficult to secrete $T e T r C B H$ I was constructed and shown to be effective in improving cellulase secretion in L. starkeyi and Y. lipolytica [26]. Recently, this approach was taken further by introducing a translational fusion partner protein and screening for cellulases that were shown to have better secretion and improved ethanol production in S. cerevisiae [35].

The enzymatic properties of CBH I expressed in yeast are basically preserved, but the observed specific activity is lower than that of most other heterologous enzymes $[27,36]$, since yeast are known to struggle to properly fold the complete CBH I protein [21, 27, 30, 36-38]. Problems with proper disulfide bond formation and "hyper glycosylation" have been proposed to affect the yield of active CBH I [27, 30, 36, 37, 39, 40]. Although, there are some reports about characterization of heterologously expressed CBH I enzymes in a specific yeast host, most are limited to some individual property relevant to that specific host, and few of them have involved systematic analysis using various approaches across a diversity of yeast. A notable exception is our prior work comparing the endoglucanase II $\operatorname{Te} T r \mathrm{CBH}$ I fusion construct in three yeasts [26]. While the goal of the fusion construct work was mainly aimed at understanding the benefits of the fusion, the results suggested significant $T e T r C B H$ I expression and activity variations between yeasts. To properly understand the differences in just the $\mathrm{CBH}$ I part in yeast, we expressed a single chimeric $\operatorname{Te} T r \mathrm{CBH}$ I gene in three oleaginous, industrially relevant yeast hosts: Y. lipolytica, L. starkeyi, and S. cerevisiae. We systematically analyzed their purified recombinant $T e T r C B H$ I by various advanced approaches and characterized these enzymes in term of active enzyme recovery, glycosylation content, thermal and proteolytic stability, surface charge difference, as well as biomass deconstruction ability.

\section{Methods \\ Cloning and expression \\ Gene cloning in E. coli}

Gene cloning in E. coli DH5-alpha strain used standard gene cloning protocols [41], in which the Gibson Assembly Cloning Kit (NEB, Ipswich, MA, USA) was employed to insert target gene into expression vectors [25]. Oligos for PCR amplification typically consisted of 16-bp overlapping regions to facilitate Gibson based cloning followed by $20-26$ bp of the region of interest.

\section{Transformation}

\section{L. starkeyi}

The chimeric T. emersonii-T. reesei cbh1 (TeTrcbh1) gene was expressed in $L$. starkeyi driven by the L. starkeyi native pyruvate kinase (pyk) promoter detailed previously [25]. Wild-type (WT) L. starkeyi NRRL Y-11557 used as the transformation host was acquired from the ARS Culture Collection (NRRL) and was transformed as previously described [25, 42-43]. Briefly, a single colony of L. starkeyi was inoculated into YPD medium and incubated at $30{ }^{\circ} \mathrm{C}$ and shaken at $225 \mathrm{rpm}$ until $\mathrm{OD}_{600}$ reached 8.0. Cells were harvested by centrifugation at $3000 \mathrm{rpm}$ for $5 \mathrm{~min}$, washed with $25 \mathrm{~mL}$ of water, and resuspended in $0.5 \mathrm{~mL}$ of $0.1 \mathrm{M} \mathrm{LiAc}$. Suspended cells $(100 \mu \mathrm{L})$ were dispensed into microcentrifuge tubes and briefly pulsed to collect the cells and remove the supernatant. The collected cells were resuspended in $240 \mu \mathrm{L}$ of PEG (50\% w/v, MW 3650), $30 \mu \mathrm{L}$ of $1 \mathrm{M} \mathrm{Li}$ acetate, $3 \mu \mathrm{L}$ of ssDNA $(10 \mu \mathrm{g} / \mu \mathrm{L})$, and linearized DNA in water to equal $27 \mu \mathrm{L}$ (300 $\mu \mathrm{L}$ total volume), and vortexed thoroughly. Typically, 5 to $8 \mu \mathrm{g}$ of linearized DNA was used per transformation. The mixture was incubated at $30{ }^{\circ} \mathrm{C}$ for $3 \mathrm{~h}$ without shaking and then heat treated at $40^{\circ} \mathrm{C}$ for $5 \mathrm{~min}$. The cells were pelleted by centrifugation for $15 \mathrm{~s}$ and the supernatant was removed. The cell pellet was resuspended in $1 \mathrm{~mL}$ of yeast extract-peptone-dextrose (YPD) medium and incubated at $30{ }^{\circ} \mathrm{C}$ and $225 \mathrm{rpm}$ for $3 \mathrm{~h}$. Cells were then centrifuged, resuspended in $0.5 \mathrm{~mL}$ of water, and plated on YPD with $30 \mu \mathrm{g} / \mathrm{mL}$ of nourseothricin (ClonNAT).

\section{Y. lipolytica}

Y. lipolytica Po1g (MatA, leu2-270, ura3-302:URA3, xpr2-332, axp-2) and the secretion vector pYLSC1 were purchased from Yeastern Biotech Co. (Taipei, Taiwan). The vector (pYLSC1, 7205 bp) contains the hybrid promoter (hp4d) and a secretion signal (XPR2 pre-region): 
atgaagctcgctaccgcctttactattctcacggccgttctggcc, which encodes the signal peptide MKLATAFTILTAVLA. This vector also contains a leucine selection marker gene (LEU2), which can complement the deletion of LEU2 gene in the parental strain of Polg. The constructs were built in the backbone of the secretion vector, pYLSC1, using the hp4d promoter. The gene coding sequence of $\operatorname{TeTrCBH}$ I was codon-optimized based on the codon bias of Y. lipolytica, and was synthesized by GenScript (described in details previously [24]). Y. lipolytica was typically grown in YPD medium.

\section{S. cerevisiae}

S. cerevisiae was grown in YPD for general growth and transformation. Transformants were grown in YNB media without uracil and containing glucose as the carbon source. S. cerevisiae was grown at $30{ }^{\circ} \mathrm{C}$ in shake flasks and shaken at $220 \mathrm{rpm}$. The CBH I expression plasmid for S. cerevisiae was built on pD1214 using a strong constitutive TEF promoter, CYC terminator, and URA3 for selection (https://www.atum.bio/products/expression -vectors/yeast). The T. reesei XYN 2 secretion signal peptide was added to the $\operatorname{Te} \operatorname{Tr} \mathrm{CBH}$ I sequence to create an expression cassette for $S$. cerevisiae (Additional file 1). This plasmid, pSc35, was transformed into BFY716 ( $S$. cerevisiae D5A ura3::APH 3' II, ura3:: $\mathrm{HPH}$ ).

Transformant colonies were assayed for activity using a $p$ NP-lactose activity assay (described below). The colony showing the most activity was selected for scale up in a 10 -L fermenter.

\section{Fermentation}

Production of $\operatorname{Te} \operatorname{TrCBH}$ I was carried out using $10 \mathrm{~L}$ of growth media in a 14-L BioFlo 310 bioreactor (New Brunswick Scientific - Eppendorf, Edison, NJ). For $L$. starkeyi and Y. lipolytica, seed cultures were inoculated from a single colony into $50 \mathrm{~mL}$ of YPD medium in a $250 \mathrm{~mL}$ flask, incubated at $30^{\circ} \mathrm{C}$ and shaken at $225 \mathrm{rpm}$. The cultures were then transferred after $24 \mathrm{~h}$ of incubation into $1 \mathrm{~L}$ of fresh YPD pH 5.0 medium in a $2.8 \mathrm{~L} \mathrm{baf-}$ fled flask. The secondary seed culture was subsequently transferred into $10 \mathrm{~L}$ of medium in the 14-L BioFlo 310 bioreactor after $\sim 36 \mathrm{~h}$ of incubation. The L. starkeyi fermentations were controlled at $30{ }^{\circ} \mathrm{C}, 300 \mathrm{rpm}$ stirring, one volume of air per volume of media per minute (VVM) at pH 5.2 in YPD medium consisting of individually made components and $5 \%$ total glucose. The fermentation was run until $\mathrm{OD}_{600}$ reached a maximum value, which was usually between 72 and $96 \mathrm{~h}$. Y. lipolytica fermentations were controlled at $\mathrm{pH} 5.0,28{ }^{\circ} \mathrm{C}, 300 \mathrm{rpm}$, and one VVM air in YPD media. Media consisted of individually made components and included citric acid and sodium citrate for increased buffering. The fermentation was run until $\mathrm{OD}_{600}$ reached maximum, usually between 72 and 120 h. S. cerevisiae fermentations were controlled at $\mathrm{pH} 5.0,30{ }^{\circ} \mathrm{C}, 300 \mathrm{rpm}$, one VVM air in YNB-ura medium with $5 \%$ total glucose. This fermentation was run for $\sim 24 \mathrm{~h}$, at which time all glucose was consumed. All culture broths were pelletized via centrifugation and concentrated using Tangential Flow Filtration (TFF) with a 10,000 MWCO membrane. The concentrated culture broths were buffer exchanged into $20 \mathrm{mM}$ Bis-Tris $\mathrm{pH}$ 6.5 in preparation for chromatographic purification.

\section{Purification}

After concentration and buffer exchange, the samples were further purified by chromatography. First the ammonium sulfate concentration of the sample was slowly adjusted to $1.5 \mathrm{M}$ and filtered with a $0.45-\mu \mathrm{m}$ Nalgene Rapid-Flow Bottle Top filter (Thermo Scientific Pierce Protein Biology Products, Rockford, IL, USA). Then the eluate was applied to a GE XK 26 column packed with hydrophobic interaction chromatography resin (Phenyl Sepharose 6 Fast Flow) and equilibrated with a $50 \mathrm{mM}$ Bis-Tris $\mathrm{pH} 6.5$ buffer containing $1.5 \mathrm{M}$ ammonium sulfate. The partially purified sample was eluted from the column with a descending ammonium sulfate gradient and desalted in $20 \mathrm{mM}$ Bis-Tris pH 6.5 buffer using two GE HiPrep 26/10 desalting columns connected in series. Next, the sample was applied to anion exchange chromatography using a Tricorn 10/100 column packed with GE Source $15 \mathrm{Q}$ resin in $20 \mathrm{mM}$ BisTris $\mathrm{pH} 6.5$ buffer and an increasing $\mathrm{NaCl}$ gradient. Final purification was done with size exclusion chromatography using a GE 26/60 Superdex 75 column eluted with $20 \mathrm{mM}$ acetate pH 5.0 buffer containing $100 \mathrm{mM} \mathrm{NaCl}$. Whenever necessary, Vivaspin $2010 \mathrm{kDa}$ concentrators were used to concentrate the samples. The desired protein fractions were identified using the $p$-nitrophenyl- $\beta$ lactoside assay [44]. All chromatography columns, resins, and concentrators were purchased from GE Healthcare (Piscataway, NJ, USA). Protein purity was assessed by

Table 1 Yield of purified and active TeTrCBH I expressed in Y. lipolvtica, L. starkeyi, and S. cerevisiae

\begin{tabular}{|c|c|}
\hline Protein & $\begin{array}{l}\text { Protein } \\
\text { yield } \\
\text { (mg/L) }\end{array}$ \\
\hline TeTrCBH I in Y. lipolvtica & 1.1 \\
\hline TeTrCBH I in L. starkeyi & 0.1 \\
\hline TeTrCBH I in S. cerevisiae peak 1 & 0.8 \\
\hline TeTrCBH I in S. cerevisiae peak 2A & 1.1 \\
\hline TeTrCBH I in S. cerevisiae peak 2B & 1.1 \\
\hline TeTrCBH I in S. cerevisiae (total of three isoforms) & 3.0 \\
\hline
\end{tabular}


SDS-PAGE and concentration was determined using $\mathrm{A}_{280}$. The values given in Table 1 are from one purification batch. Y. lipolytica and L. starkeyi TeTrCBH I enzymes were purified several times with similar results. Only one purification batch was needed for S. cerevisiae TeTrCBH I. $T$. reesei $\mathrm{CBH}$ I ( $\operatorname{Tr} \mathrm{CBH}$ I) was cloned, expressed, and purified as described before [24].

\section{High performance liquid chromatography}

To verify the purity of the chimeric CBH I enzymes, HPSEC with TSK gel G3000SWXL column (Tosoh Bioscience, Tokyo, Japan) was used. Both UV (at $280 \mathrm{~nm}$; Rainin Dynamax UV-D II) and RI (Jasco, A029660872) detectors were used to obtain information about glycosylation differences. One hundred $\mu \mathrm{L}$ of approximately $2 \mathrm{mg} / \mathrm{mL}$ protein sample were loaded onto the HPSEC column at a flow rate of $0.5 \mathrm{~mL} / \mathrm{min}$ in $20 \mathrm{mM}$ acetic acid pH 5 with $100 \mathrm{mM} \mathrm{NaCl}$.

\section{Proteolytic stability}

Protein samples at a concentration of $0.25 \mathrm{mg} / \mathrm{mL}$ were incubated with Thermolysin (Promega, Madison, USA) or $\alpha$-chymotrypsin (Promega, Madison, USA) at $25 \mu \mathrm{g} /$ $\mathrm{mL}$ or $4 \mu \mathrm{g} / \mathrm{mL}$, respectively, in a volume of $200 \mu \mathrm{L}$ for $0.5 \mathrm{~h}, 1 \mathrm{~h}, 2 \mathrm{~h}$, and $4 \mathrm{~h}$. Thermolysin and $\alpha$-chymotrypsin reactions were stopped by addition of 1\% EDTA (SigmaAldrich, St. Louis, USA) and a protease inhibitor cocktail (Sigma-Aldrich, St. Louis, USA), respectively. All samples, including the time zero $\left(t_{0}\right)$ point were loaded on a NuPAGE Bis-Tris Gel (Thermo Fisher Scientific Inc., Rockford, USA) for electrophoresis and densitometry analyses, performed with Image J [45].

\section{Thermal stability}

Thermal stability was measured using a Microcal model VP-DSC calorimeter (Microcal, Inc., Northampton, USA). Data analysis was completed by Origin for DSC software (OriginLab, Northampton, USA). Samples were diluted up to $100 \mu \mathrm{g} / \mathrm{mL}$ protein in $20 \mathrm{mM}$ acetate $\mathrm{pH} 5.0$ buffer with $100 \mathrm{mM} \mathrm{NaCl}$. The calorimeter scan rate was $60{ }^{\circ} \mathrm{C} / \mathrm{h}$ over a range of $25-80^{\circ} \mathrm{C}$.

\section{Comparative SDS-PAGE}

Protein samples were diluted in $4 \times$ LDS sample buffer (Life Technologies Corp., Carlsbad, USA) and incubated at $95{ }^{\circ} \mathrm{C}$ for $10 \mathrm{~min}$ prior to loading on a NuPAGE BisTris Gel (Thermo Fisher Scientific Inc., Rockford, USA). Electrophoresis was conducted in MOPS (3-(N-morpholino) propanesulfonic acid) buffer and $200 \mathrm{~V}$ for $50 \mathrm{~min}$. Coomassie staining was performed using Thermo Scientific ${ }^{\mathrm{TM}}$ Pierce $^{\mathrm{TM}}$ Power Stainer (Thermo Fisher Scientific Inc., Rockford, USA), whereas staining for glycosylation was done using the Pierce ${ }^{\mathrm{TM}}$ Glycoprotein Staining
Kit (Thermo Fisher Scientific Inc., Rockford, USA), following the manufacturer's instructions. Western-Blot analyses were performed as reported earlier [46].

\section{Isoelectric focusing}

A Novex pH 3-7 IEF Protein Gel system (Invitrogen, NY) was used for isoelectric focusing (IEF) experiments using manufacturer recommended protocols and IEF Markers pH 3-10 (Invitrogen, NY). Approximately $80 \mu \mathrm{g}$ of protein concentrate was loaded into each well.

\section{Digestion assays}

Cellulase activity was measured using dilute-acid-pretreated corn stover (PCS) as a substrate. Specifically, the PCS used was NREL dilute-acid-pretreated corn-stover P050921, produced in a vertical pulp digester supplied by Sunds Defibrator (now Metso Corporation, Helsinki, Finland) as described earlier [47], with a residence time of approximately one min at $190{ }^{\circ} \mathrm{C}$ and $0.45 \mathrm{~g} \mathrm{H}_{2} \mathrm{SO}_{4}$ per $\mathrm{g}$ dry biomass at $30 \%$ solids loading. These conditions yielded a material having $59.1 \%$ glucan, $5.1 \%$ xylan, and $25.3 \%$ lignin. Because the theoretical molecular weight of the $\operatorname{Te} T r \mathrm{CBH} \mathrm{I}$ is slightly greater than that of the $\operatorname{Tr} \mathrm{CBH}$ $\mathrm{I}$, an equal molar loading resulted in a loading of $25.0 \mathrm{mg}$ enzyme/g cellulose for $T e T r C B H ~ I$, versus $24.6 \mathrm{mg}$ enzyme/g cellulose for $\operatorname{TrCBH}$ I. This equals a loading of $5.7 \mathrm{mg}$ enzyme/mL PCS. In addition to purified $T e T r C B H$ I enzymes, each assay also contained the catalytic domain of Acidothermus cellulolyticus E1 endoglucanase (with the enhanced Y245G mutation [48]) loaded at $1.7 \mathrm{mg}$ enzyme/g cellulose, along with Aspergillus niger beta-glucosidase (BGL). BGL was chromatographically purified from the commercial mixture Novozyme 188 (Novozymes North America, Franklinton, NC, USA) and was loaded into the reaction mixtures at a concentration of $0.4 \mathrm{mg}$ enzyme/g of cellulose substrate.

Assays were carried out at $40{ }^{\circ} \mathrm{C}$ in $20 \mathrm{mM}$ acetate $\mathrm{pH}$ 5.0 buffer containing $0.02 \%(\mathrm{w} / \mathrm{v})$ sodium azide to inhibit microbial growth. Assays were performed in triplicate using initial digestion volumes of $1.7 \mathrm{~mL}$ in crimp-sealed 2.0-mL HPLC vials which were constantly mixed by inversion (10 revolutions per min) in a $40{ }^{\circ} \mathrm{C}$ water bath. At designated time points during the digestions, representative $0.1-\mathrm{mL}$ aliquots of liquid and solids were withdrawn for analysis. These aliquots were diluted 18 -fold with deionized water into sealed 2.0-mL HPLC vials and immersed for $10 \mathrm{~min}$ in a boiling water bath to terminate the enzymatic reactions. The diluted aliquots were then filtered through $0.2-\mu \mathrm{m}$ filters before determination of released sugars by HPLC, as described previously [24]. 


\section{Results and discussion}

The TeTrcbh1 fusion gene was expressed in Y. lipolytica, L. starkeyi, and S. cerevisiae. The recombinant TeTrCBH I enzymes were purified and used for further characterization. Our prior work with the $\mathrm{CBH}$ I-endoglucanase II fusion protein showed variability in the active protein expression levels and in biomass deconstruction efficacy when expressed in different yeast species [26]. To better understand the basis of these differences in $\mathrm{CBH}$ I performance, without interference from the fusion partner, we planned a series of experiments investigating the yields of purified and active $\operatorname{TeTrCBH}$ I enzyme, sample heterogeneity (e.g., glycosylation and charge differences), thermal and proteolytic stability, and performance. These experiments were performed with $T e \operatorname{Tr} \mathrm{CBH}$ I expressed in Y. lipolytica, L. starkeyi, and S. cerevisiae to better understand the behavior of this fusion construct across relevant oleaginous yeast species.

\section{Identification and recovery of TeTrCBH I expressed in $Y$. lipolytica, L. starkeyi, and S. cerevisiae}

The purified recombinant proteins from the three yeast species were identified by SDS-PAGE and Western blot (Fig. 1). First, we compared the recovery of purified and active $\operatorname{TeTr} \mathrm{CBH}$ I from Y. lipolytica, L. starkeyi, and S. cerevisiae (Table 1). By only collecting fractions displaying activity on $p$ NP-lactose [27], the purity of the enzymes studied was enhanced and this assured that the proteins we purify were active. L. starkeyi cultures had the lowest recovery of purified and active $\operatorname{Te} T r \mathrm{CBH}$ I $(0.1 \mathrm{mg} / \mathrm{L})$, followed by $Y$. lipolytica $(1.1 \mathrm{mg} / \mathrm{L})$, representing a greater than tenfold increase in titer. The low recovery of active enzyme from $L$. starkeyi agrees with our earlier work with the CBH I-endoglucanase II fusion protein [26], where the fusion construct was necessary to improve the L. starkeyi TeTrCBH I yield. The S. cerevisiae enzyme was recovered at an even higher yield $(3.0 \mathrm{mg} / \mathrm{L})$, which is more than a 30-fold increase. These results are generally consistent with previously reported enzyme yield values [37, 39, 49]. However, the recovery of $1.1 \mathrm{mg}$ enzyme/L for $Y$. lipolytica is much lower than previously reported, which is based on total secreted protein yield (i.e., $24 \mathrm{mg} / \mathrm{L}$ ) [24]. The total secreted protein yield reported by this earlier study was based on Western blot densitometry and likely includes partially degraded, misfolded, incorrectly modified, and/or inactive enzymes. These data are further examples that demonstrate the low yields of active CBH I that are typically purified from yeast. Heterogeneity from possible misfolding, proteolysis, and modification appears to be considerable when $T e T r C B H$ I is expressed in S. cerevisiae, where the production level is almost threefold higher than in Y. lipol$y$ tica. However, in this case there were three distinct and active $\operatorname{Te} \operatorname{Tr} \mathrm{CBH} \mathrm{I}$ isoforms (Additional file 2). The different isoforms first appeared during anion exchange chromatography indicating possible surface charge differences and were further separated by SEC. Closer inspection of the SEC peaks showed that all three peaks were indeed of slightly different size (Fig. 1 and Additional file 2). This finding strongly suggests that $S$. cerevisiae expresses active $T e T r C B H$ I variants which differ in surface charge and size. These results can be explained by folding issues, but given that the enzymes are active, variability in glycosylation is also indicated. Glycan decoration of proteins impacts the surface charge and can significantly alter the protein size.

\section{Extent of glycosylation and heterogeneity}

To assess the heterogeneity and the extent of glycosylation of the chimeric $\operatorname{Te} T r \mathrm{CBH}$ I enzymes produced

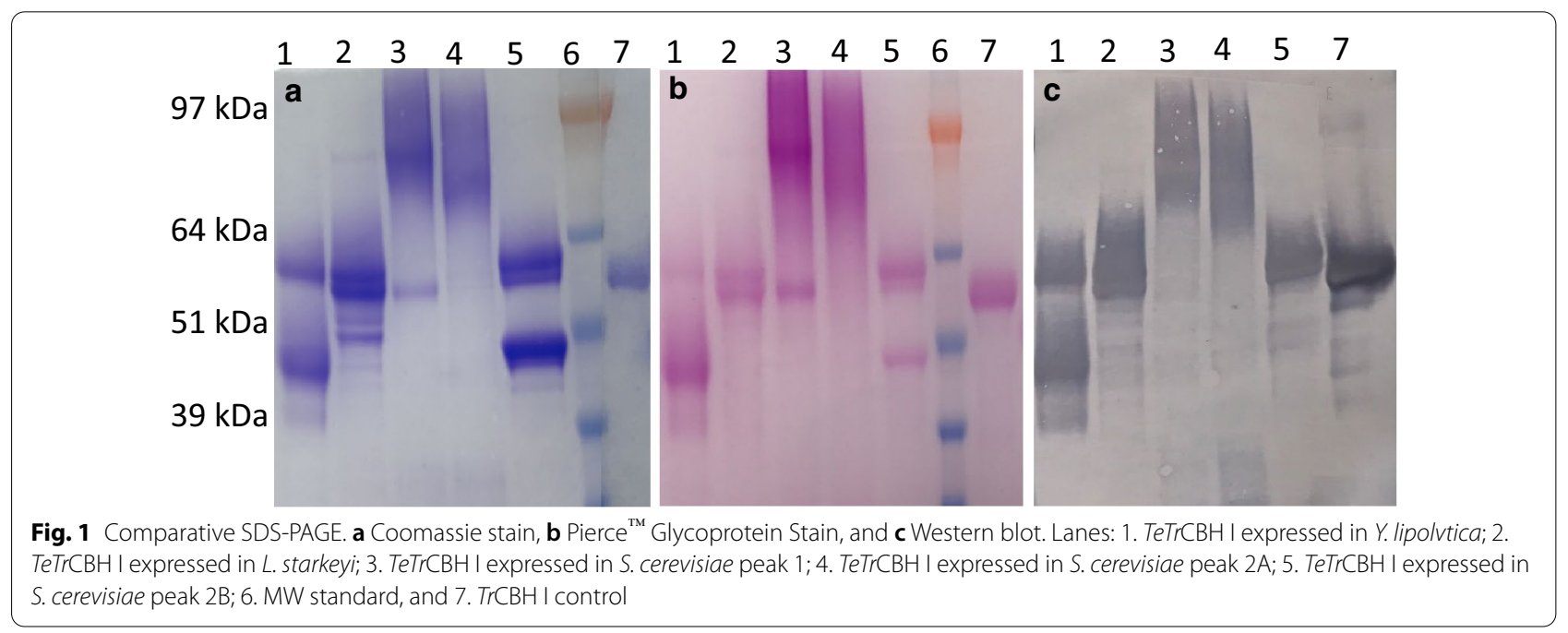


Table 2 HPSEC peak mobility and RI/UV ratios. Higher numbers for the RI/UV area ratio indicate higher glucan content. HPSEC graphs in Additional file 2

\begin{tabular}{llllll}
\hline & $\begin{array}{l}\text { Rl peaks mobility } \\
\text { (minutes) }\end{array}$ & $\begin{array}{l}\text { RI combined } \\
\text { peak area }\end{array}$ & $\begin{array}{l}\text { UV } \\
\text { peaks mobility (minutes) }\end{array}$ & $\begin{array}{l}\text { UV combined } \\
\text { peak area }\end{array}$ & $\begin{array}{l}\text { RI/UV } \\
\text { area ratio }\end{array}$ \\
\hline TrCBH I & $15.12,18.68$ & 1202 & 14.95 & 2638 & 0.46 \\
TeTrCBH I in S. cerevisiae peak 1 & $13.30,20.42$ & 1625 & 13.25 & 2424 & 0.67 \\
TeTrCBH I in S. cerevisiae peak 2A & $13.10,19.42$ & 1487 & 13.08 & 2260 & 0.66 \\
TeTrCBH I in S. cerevisiae peak 2B & $18.23,21.20$ & 1130 & $17.33,18.57$ & 2848 & 0.40 \\
TeTrCBH I in Y. lipolytica & $15.07,19.97$ & 1130 & $14.92,18.45,21.18$ & 2639 & 0.43 \\
TeTrCBH I in L. starkeyi & $15.18,19.20$ & 1525 & $15.15,21.23$ & 2486 & 0.61 \\
\hline
\end{tabular}

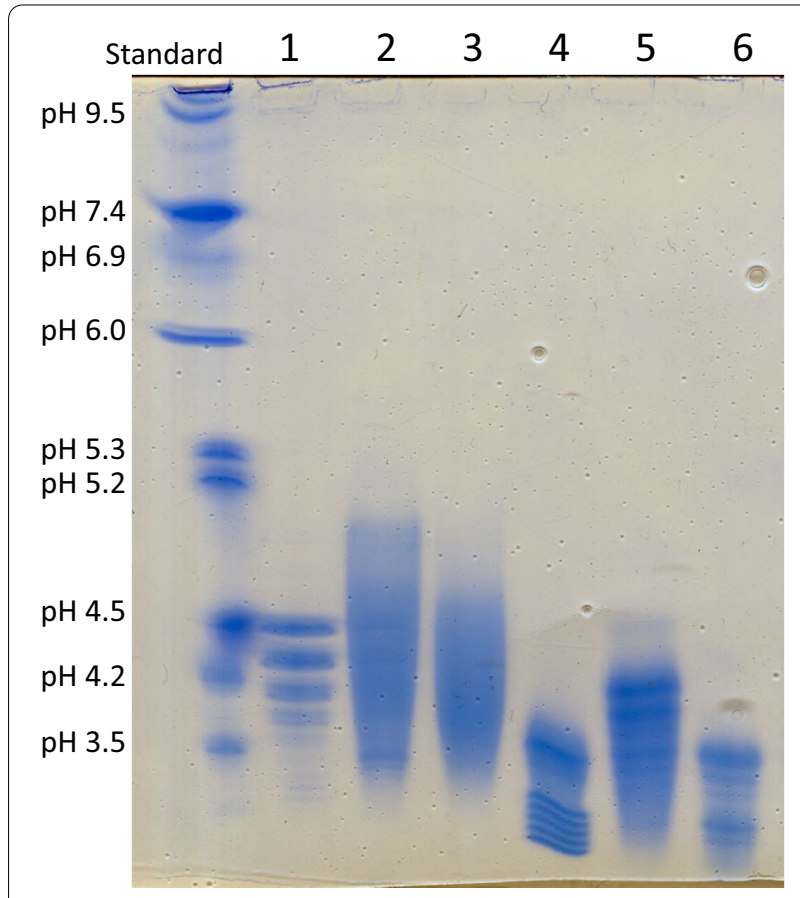

Fig. 2 Isoelectric focusing gel. Lanes: 1. TrCBH I; 2. TeTrCBH I expressed in S. cerevisiae peak 1; 3. TeTrCBH I expressed in S. cerevisiae peak 2A; 4. TeTrCBH I expressed in S. cerevisiae peak 2B; 5. TeTrCBH I expressed in Y. lipolvtica; 6. TeTrCBH I expressed in L. starkeyi. Standards: cytochrome C (10.7 pl), ribonuclease A (9.5 pl), lectin (8.3, 8.0, $7.8 \mathrm{pl})$, myoglobin $(7.4,6.9 \mathrm{pl})$, carbonic anhydrase (6.0 pl), $\beta$-lactoglobulin (5.3, $5.2 \mathrm{pl})$, trypsin inhibitor (4.5 pl), glucose oxidase (4.2 pl), amyloglucosidase (3.5 pl)

in the three yeast, several methods were used including HPSEC (Table 2), SDS-PAGE with Coomassie blue staining (Fig. 1a), glycosylation staining (Fig. 1b), and Western blot analysis (Fig. 1c), and isoelectric focusing (Fig. 2). All of the TeTrCBH I samples tested showed multiple apparent MW bands in the Western blot and glycosylation stained PAGE, indicating degradation and/or variable glycosylation extent. Curiously, some of the predominant bands were of low apparent molecular weight. This is especially true for the $\operatorname{Te} \operatorname{TrCBH}$ I expressed in $Y$. lipolytica. To verify and quantify all sample components, we analyzed them with HPSEC and compared the results to $\operatorname{TrCBH} \mathrm{I}$ expressed in $T$. reesei (Table 2, HPSEC graphs in Additional file 2).

\section{Sample purity, degradation, and glycosylation}

The HPSEC results agreed with the SDS-PAGE analysis and showed multiple peaks (Table 2, HPSEC graphs in Additional file 2) for $\operatorname{Te} \operatorname{TrCBH}$ I expressed in each yeast species. Furthermore, HPLC with both RI and UV detectors allowed reliable measurements of the contents of each sample. The main peaks for all samples, except peak 2B of $\operatorname{Te} \operatorname{Tr} \mathrm{CBH}$ I expressed in S. cerevisiae, had similar retention times to $\operatorname{TrCBH} \mathrm{I}$; with only minor or insignificant secondary peaks (Fig. 1a, c). Degradation can be expected to show up as separate peaks in chromatography and as distinct bands in SDS-PAGE, whereas variable glycosylation results in broad peaks and smeared bands. S. cerevisiae $\operatorname{Te} \operatorname{Tr} \mathrm{CBH}$ I peak $2 \mathrm{~B}$ had two major peaks with significantly longer retention values and two "sharp" bands in SDS-PAGE, indicating significant degradation and low extents of glycosylation. The other two S. cerevisiae $\operatorname{Te} \operatorname{TrCBH}$ I samples, peak 1 and peak $2 \mathrm{~A}$, eluted as single peaks, but were of much higher apparent molecular weight and appeared as elongated and smeared SDSPAGE bands, suggesting a high glycan content.

\section{Western blot and sample degradation}

The Western blot results (Fig. 1c) mostly agreed with the Coomassie stained PAGE (Fig. 1a), showing only minor bands that were not visible in Western blot. This result is expected, because our purification protocol selects for active fractions. Inactive fractions can only be fractionated if they co-elute with active forms of the enzyme. S. cerevisiae peak 1 and peak $2 \mathrm{~B}$ were the most notable exceptions. Both fractions had low or lower molecular weight bands that were barely visible. It is possible that 
these species are unrelated impurities; however, the $S$. cerevisiae peak 1 is more likely to be a low concentration contamination from peak $2 \mathrm{~B}$ that was not fully separated during purification. In the case of enzymes expressed from all yeast hosts studied, Western blot stained bands can be observed which verifies CBH I content. If degraded forms of CBH I have a weaker Western blot signal, both species can be assumed to be CBH I. This could be the case especially if a significant region, such as the CBM1 domain (and the linker peptide), is lost. The lower molecular weight SDS-PAGE bands of $S$. cerevisiae peak 2B and Y. lipolyctica could reflect this outcome (Fig. 1). Overall, the SDS-PAGE results for yeast expressed

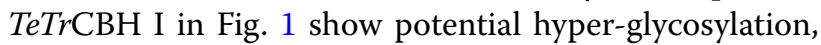
degradation, and double bands compared to the T. reesei expressed $\operatorname{Tr} \mathrm{CBH} \mathrm{I}$, again demonstrating the problems yeast have with $\mathrm{CBH}$ I expression and stability.

\section{Glycosylation}

Using both UV and RI detectors in HPSEC allowed us to quantify the extent of glycosylation as an RI/UV peak area ratio due to the insignificant absorption of glycosylation at $280 \mathrm{~nm}$, whereas it is readily detectable by the RI-detector. Higher numbers for the RI/UV area ratio indicate a higher glycan content. As expected from our previous results, S. cerevisiae $\operatorname{Te} T r \mathrm{CBH}$ I peak 2B and Y. lipolytica TeTrCBH I have a lower glycan content (Table 2). Also, S. cerevisiae TeTrCBH I peak 2B has clear signs of degradation (two major peaks), whereas $Y$. lipolyctica TeTrCBH I shows two minor peaks in HPSEC. In contrast, $T$. reesei $\operatorname{TrCBH}$ I migrates as a single band in SDS-PAGE (Fig. 1a). Clearly, T. reesei expression produces intact $\operatorname{TrCBH}$ I with a relatively low extent of glycosylation, which contrasts with yeast which secrete a highly glycosylated $\operatorname{Te} \operatorname{Tr} \mathrm{CBH}$ I. The RI/UV ratio of $S$. cerevisiae TeTrCBH I peaks 1 and $2 \mathrm{~A}$ show a wide range of glycosylation extent, which agrees well with the glycan stained PAGE shown in Fig. 1b. Both species show no bands below the molecular weight of CBH I expressed in $T$. reesei, suggesting that glycosylation might protect the enzyme from degradation. This is clearly demonstrated in the case of $Y$. lipolyctica $\operatorname{Te} \operatorname{TrCBH} \mathrm{I}$, which appears to be less glycosylated by PAGE analysis than the L. starkeyi TeTrCBH I and on Western Blot migrates as a main band below $50 \mathrm{kDa}$ (Fig. 1c). These results strongly suggest that Y. lipolyctica TeTrCBH I is somewhat degraded. If the difference in molecular weight relates to the loss of the CBM1 and linker regions, this result could indicate that $Y$. lipolyctica has difficulty in adequately $O$-glycosylating the linker peptide region which is consequently degraded.

\section{Surface charge differences}

To further characterize the physical differences between these enzymes, we performed isoelectric focusing (IEF, Fig. 2). IEF produced a unique isoelectric point distribution for each enzyme from the three yeast species tested. The results agreed with the overall extent of glycosylation. Interestingly, both S. cerevisiae $\operatorname{Te} T r \mathrm{CBH}$ I peak $2 \mathrm{~B}$ and L. starkeyi TeTrCBH I had isoelectric point distributions at a particularly low $\mathrm{pH}$. Overall, these results show a significant variability of surface charge which correlates to the difference in glycosylation extent among the samples. Enzymes produced by these two yeast clearly have different glycosylation content compared to the other yeast expression products and $\operatorname{Tr} \mathrm{CBH} \mathrm{I}$, perhaps a consequence of the differences in their protein glycosylation pathways. This concept has been widely reported in literature, particularly in relation to the variability in the extent and complexity of $\mathrm{N}$-glycosylation of secreted proteins [50].

\section{Thermal and proteolytic stability}

Glycosylation has been reported to strongly affect cellulase stability [51]. To understand the potential effects of glycosylation on performance, we performed thermal and proteolytic stability experiments comparing the $\operatorname{Te} \operatorname{Tr} \mathrm{CBH}$ I produced in yeast to $\operatorname{Tr} \mathrm{CBH}$ I expressed in T. reesei. All the yeast-expressed $\operatorname{Te} T r \mathrm{CBH}$ I chimeras, except that expressed in Y. lipolytica, had a similar thermal stability with a $T_{\max }$ close to $65^{\circ} \mathrm{C}$, which is the value measured for $\operatorname{Tr} \mathrm{CBH}$ I (Table 3). A significantly lower $T_{\max }\left(61.8^{\circ} \mathrm{C}\right)$ was measured for $Y$. Lipolytica $T e T r C B H$ I. This result is consistent with the notion that glycosylation, and especially $O$-glycosylation, is often correlated to thermal stability of cellulases and other proteins [52-54]. When considered together, the increased degradation (Fig. 1), low extent of glycosylation, and unique surface charge properties (Fig. 2) of Y. lipolytica TeTrCBH I suggest that the glycosylation of these proteins differ when compared to the native enzyme and that this could be the

\begin{tabular}{|c|c|}
\hline Protein & $\mathrm{T}_{\max }\left({ }^{\circ} \mathrm{C}\right)$ \\
\hline $\operatorname{TrCBHI}$ & $65.1 \pm 0.9$ \\
\hline TeTrCBH I in Y. lipolytica & $61.8 \pm 0.7$ \\
\hline TeTrCBH I in L. starkeyi & $64.9 \pm 0.2$ \\
\hline TeTrCBH I in S. cerevisiae peak 1 & $65.7 \pm 0.3$ \\
\hline TeTrCBH I in S. cerevisiae peak 2A & $65.6 \pm 0.3$ \\
\hline TeTrCBH I in S. cerevisiae peak 2B & $64.9 \pm 0.5$ \\
\hline
\end{tabular}




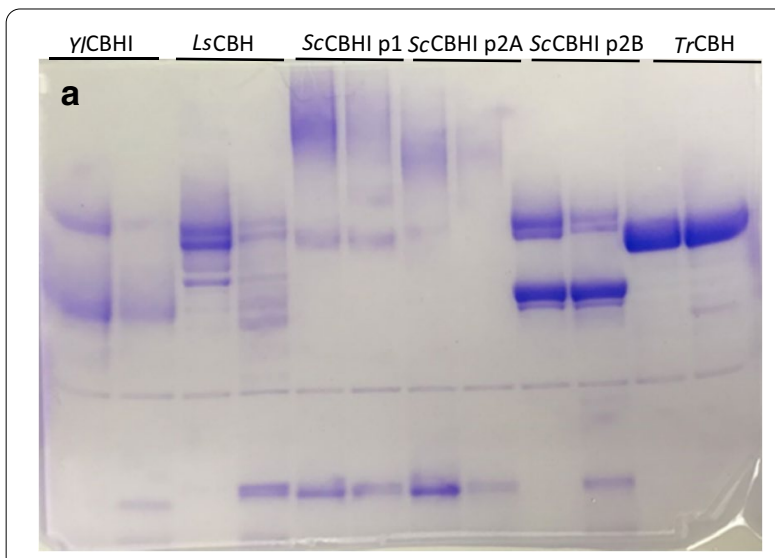

т0 $0.5 \mathrm{~h}$ т0 $0.5 \mathrm{~h}$ т0 $0.5 \mathrm{~h}$ т0 $0.5 \mathrm{~h}$ т0 $0.5 \mathrm{~h}$ т0 $0.5 \mathrm{~h}$

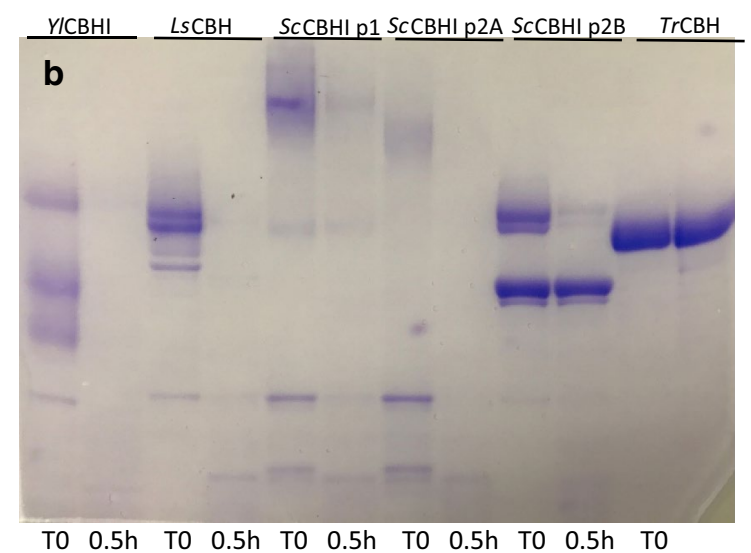

Fig. 3 a Thermolysin treatment. TO: initial sample before incubation. $0.5 \mathrm{~h}$ : Sample after 0.5 -h incubation. $\mathbf{b}$ a-chymotrypsin treatment. T0: initial sample before incubation. $0.5 \mathrm{~h}$ : Sample after 0.5 -h incubation

reason for the lower thermal stability shown by the heterologous enzyme.

Ttreatment with thermolysin (Fig. 3a) and $\alpha$-chymotrypsin (Fig. 3b) showed very little degradation for $\operatorname{TrCBH}$ I, whereas all yeast-expressed $\operatorname{Te} \operatorname{TrCBH} \mathrm{I}$ enzymes showed significant loss of protein. Interestingly, the lower molecular weight peak of S. cerevisiae peak 2B was not affected by proteases and was of similar size as the Y. lipolytica and L. starkeyi $\operatorname{TeTr} \mathrm{CBH}$ I enzymes after proteolysis treatment. This species, therefore, is likely a degraded form of the CBH I catalytic module that cannot be further cleaved by these enzymes. Clearly, yeast expressed $\operatorname{Te} \operatorname{Tr} \mathrm{CBH}$ I has lower proteolysis stability, supporting previous results, where $\mathrm{CBH} I$ expression is enhanced by expressing them in host yeast with protease gene deletions.

\section{Biomass deconstruction assays}

The three purified chromatographic forms of S. cerevisiae expressed $\operatorname{Te} \operatorname{TrCBH}$ I were evaluated for PCS cellulose degradation and compared to $\operatorname{Tr} \mathrm{CBH}$ I purified from its native host, T. reesei (Fig. 4). All three forms of the $S$. cerevisiae $\operatorname{Te} \operatorname{Tr} \mathrm{CBH}$ I showed a significantly lower extent of cellulose conversion compared to $\operatorname{TrCBH}$ I. Surprisingly, peak $2 \mathrm{~B}$ performed similarly to peak 1 despite being degraded into two peaks according to HPLSEC analysis (Additional file 2: Fig. S5). In contrast, peak 2A performed markedly worse. Thus, in this case, the degradation of peak 2B does not appear to have negatively impacted activity, highlighting the different performance of the various fractions derived from the same enzyme.

The chimeric $T e T r C B H$ I purified from L. starkeyi and Y. lipolytica converted $70 \%$ of the available PCS cellulose compared to $80 \%$ conversion for the $\operatorname{TrCBH}$ I purified from its native host (Fig. 5). Note that peak 1 from S. cerevisiae converted only $60 \%$ of the substrate. Despite taking the best fraction for S. cerevisiae expressed $T e T r C B H$ I, it still underperformed compared to the L. starkeyi and $Y$. lipolytica expressed chimeric $\operatorname{Te} \operatorname{Tr} \mathrm{CBH}$ I. However, $\operatorname{TrCBH}$ I clearly is more active than the L. starkeyi and $Y$. lipolytica expressed $\operatorname{Te} \operatorname{Tr} \mathrm{CBH}$ I chimeras shown by reaching a conversion of over $80 \%$ in $100 \mathrm{~h}$, compared to about $70 \%$ for the other two enzymes. These results show that there are significant $\operatorname{Te} \operatorname{Tr} \mathrm{CBH}$ I activity differences between the three yeast, and that $Y$. lipolytica is the best host for stability and activity.

Our results agree with previous observations of lower CBH I enzyme activity when expressed in yeast [27, 36]. However, the significant activity differences between chimeric $T e T r C B H$ I fractions isolated from one S. cerevisiae expression batch is new. These not only enrich our knowledge of the $\mathrm{CBH}$ I activity diversity expressed in yeast, but also reveals the complexities of $\mathrm{CBH}$ I expression in yeast. Our results show that the causes of this variability can be from differences in the genetic background of the yeast hosts which leads to folding, glycosylation, and proteolytic stability differences.

While yields and stability of heterologously expressed enzymes are important characteristics, it is crucial that the enzymes produced are sufficiently active to perform the expected function. For CBP, the hydrolysis of cellulose at a rate sufficient to permit cell growth is essential. Our study provides fundamental information about chimeric $\operatorname{Te} \operatorname{Tr} \mathrm{CBH}$ I expressed in different yeast through systematical analysis. This deeper understanding is necessary to enable enhanced CBH I expression in yeast, thus helping to create new CBP yeast with efficient lignocellulose degradation capability.

\section{Conclusions}

We report studies of the heterologous expression, biochemical and catalytic properties, and biomass decon-

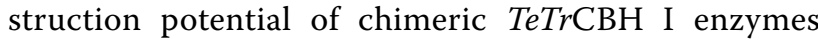




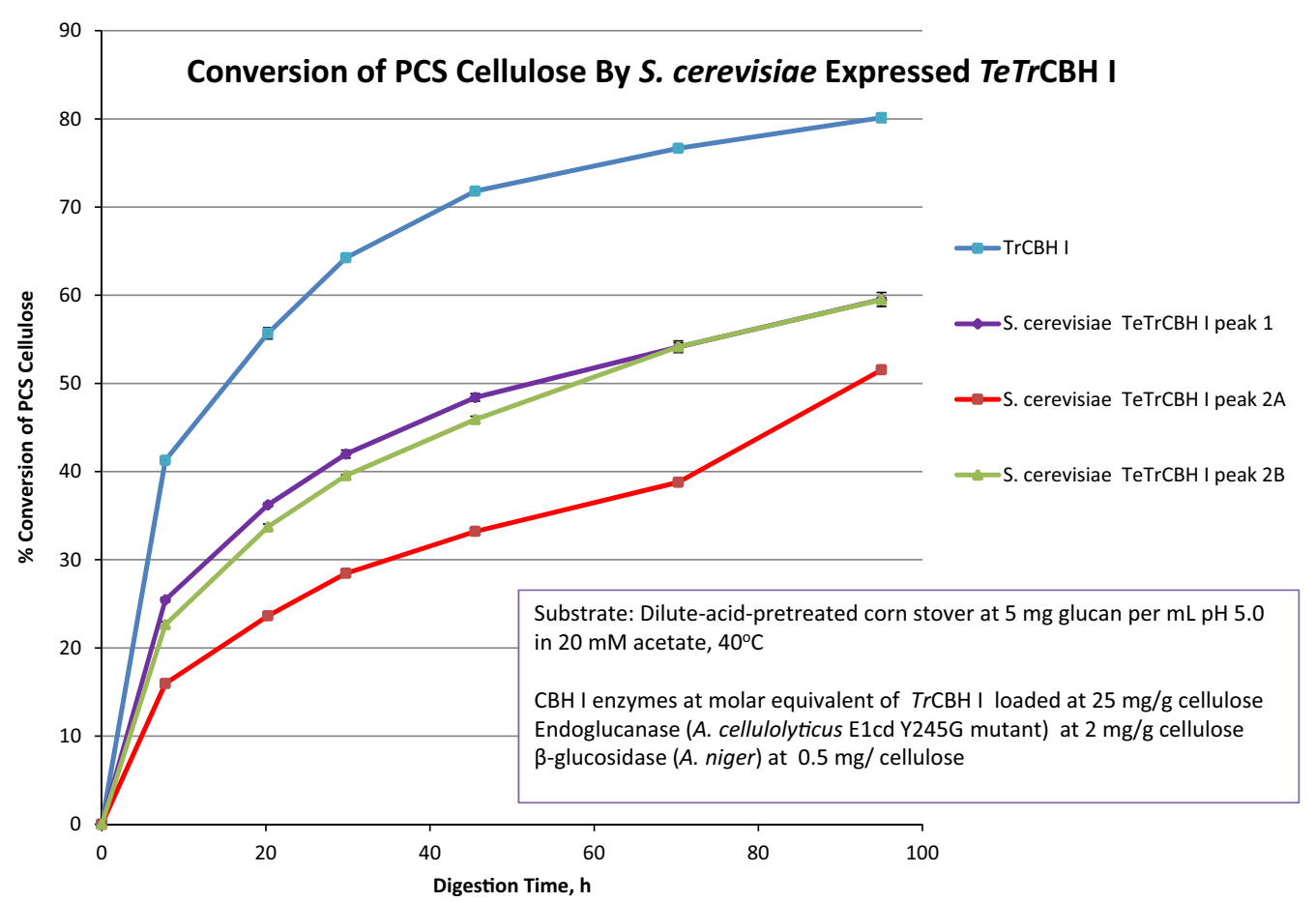

Fig. 4 Conversion of PCS cellulose by TeTrCBH I enzymes purified from S. cerevisiae and T. reesei

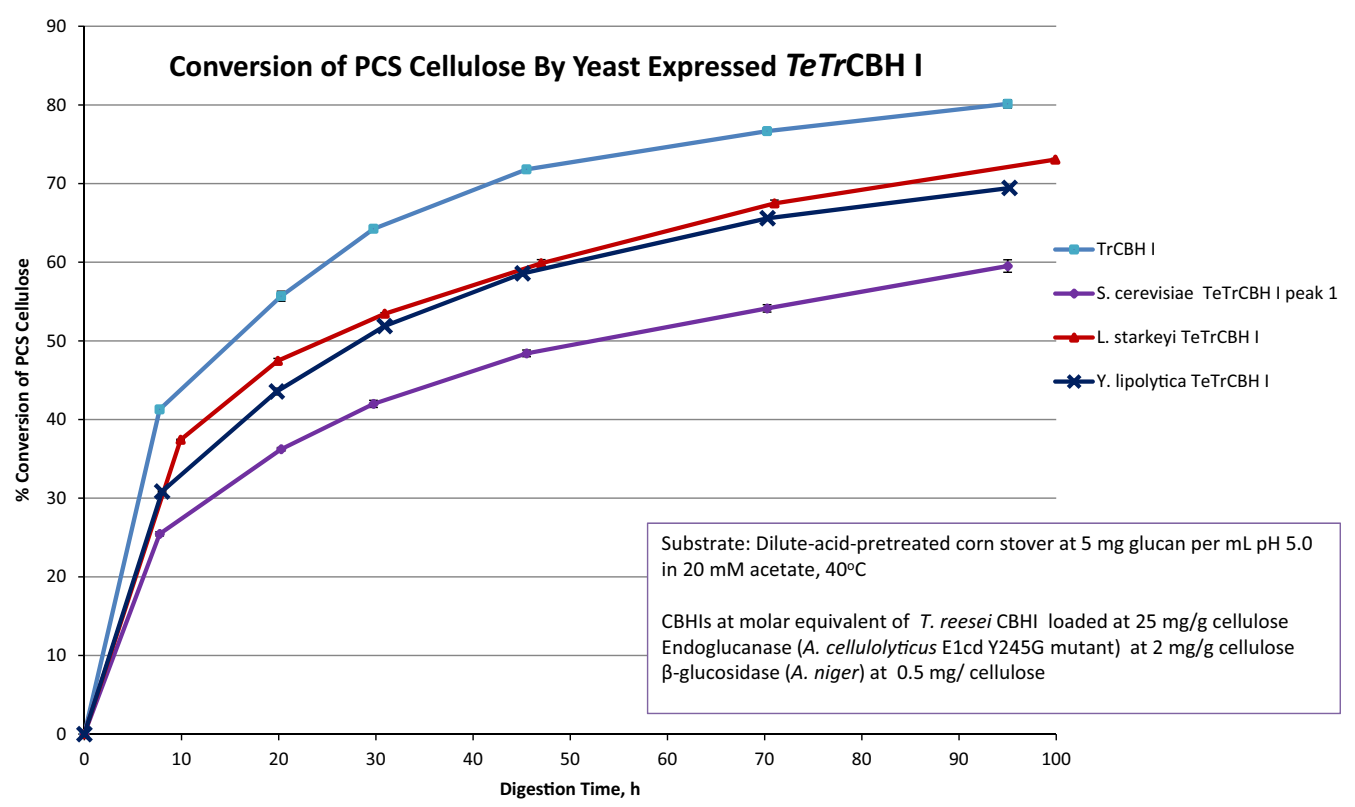

Fig. 5 Conversion of PCS cellulose by TeTrCBH I enzymes purified from S. cerevisiae, L. starkeyi, Y. lipolytica, and T. reesei

expressed in three different, oleaginous, industrially relevant yeast: Y. lipolytica, L. starkeyi, and S. cerevisiae. Yields of purified active $T e \operatorname{TrCBH}$ I, sample heterogeneity (glycosylation and charge differences), thermal and proteolytic stability, and performance were determined to better understand cellulase expression in yeast. We show that $S$. cerevisiae demonstrates the highest yield of active $\operatorname{Te} \operatorname{TrCBH}$ I construct; however, this enzyme 
appears to be the lowest in performance capability on a biomass substrate (Fig. 5). The L. starkeyi and Y. lipolytica expressed chimeric TeTrCBH I enzymes have comparable activity, which is also much higher than that of the $S$. cerevisiae produced enzyme. Y. lipolytica produces over ten times more purified and active $T e T r C B H$ I enzyme than L. starkeyi (Table 1). Clearly, L. starkeyi and $S$. cerevisiae are inferior CBP candidates compared to $Y$. lipolytica. Based on these results, Y. lipolytica could, therefore, perform adequately in CBP from the perspective of producing sufficiently active CBH I; however, the available enzyme titers may not be high enough to function in an industrial setting $[22,23]$.

\section{Supplementary Information}

The online version contains supplementary material available at https://doi. org/10.1186/s13068-020-01856-z.

Additional file 1. Schematic representation of the $\operatorname{Te} \operatorname{Tr} C B H$ I expression construct in S. cerevisiae.

Additional file 2. High performance size exclusion chromatography UV and RI chromatograms.

\section{Abbreviations}

CBP: Consolidated bioprocessing; T. reesei: Trichoderma reesei; S. cerevisiae: Saccharomyces cerevisiae; Y. lipolytica: Yarrowia lipolytica; L. starkeyi: Lipomyces starkeyi; CBH I: Cellobiohydrolase I; $\operatorname{TrCBH~I:~T.~reesei~CBH~I;~TrEG~II:~T.~reesei~}$ EG II; TeTrCBH I: A chimeric CBH I generated by the fusion of the catalytic module from Talaromyces emersonii CBH I with the linker peptide and cellulosebinding module from $\mathrm{T}$. reesei $\mathrm{CBH}$ I; $\mathrm{VWM}$ : Volume of gas per volume of media per minute; PCS: Dilute-acid-pretreated corn stover; HPSEC: High-performance size exclusion chromatography; SDS-PAGE: Sodium dodecyl sulfate-polyacrylamide gel electrophoresis.

\section{Acknowledgements}

This work was authored by the National Renewable Energy Laboratory, operated by Alliance for Sustainable Energy, LLC, for the U.S. Department of Energy (DOE) under Contract No. DE-AC36-08GO28308. Funding provided by U.S. Department of Energy Office of Energy Efficiency and Renewable Energy Bioenergy Technologies Office. The views expressed in the article do not necessarily represent the views of the DOE or the U.S. Government. The U.S. Government retains and the publisher, by accepting the article for publication, acknowledges that the U.S. Government retains a nonexclusive, paid-up, irrevocable, worldwide license to publish or reproduce the published form of this work, or allow others to do so, for U.S. Government purposes.

\section{Author contributions}

All authors read and approved the final manuscript. All authors have contributed to the drafting and editing of the manuscript.

\section{Funding}

Funding provided by U.S. Department of Energy Office of Energy Efficiency and Renewable Energy Bioenergy Technologies Office. We thank the Bioenergy Technologies Office for funding under Contract DE-AC36-08GO28308 with the National Renewable Energy Laboratory.

Availability of data and materials The datasets used and/or analyzed during the current study are available from the corresponding author on reasonable request. All data generated or analyzed during this study are included in this manuscript and additional files.

\section{Ethics approval and consent to participate}

Not applicable.

\section{Consent for publication}

All authors have given their consent for the publication.

\section{Competing interests}

The authors declare that they have no competing interests.

Received: 25 September 2020 Accepted: 10 December 2020

Published online: 06 January 2021

\section{References}

1. Lynd LR, Weimer PJ, Willem HVZ, Pretorius IS. Microbial cellulose utilization: fundamentals and biotechnology. Microbiol Mol Biol Rev. 2002;66:506-77.

2. Cragg SM, Beckham GT, Bruce NC, Bugg TD, Distel DL, Dupree P, Etxabe AG, Goodell BS, Jellison J, McGeehan JE, et al. Lignocellulose degradation mechanisms across the Tree of Life. Curr Opin Chem Biol. 2015;29:108-19.

3. Mathews SL, Pawlak J, Grunden AM. Bacterial biodegradation and bioconversion of industrial lignocellulosic streams. Appl Microbiol Biotechnol. 2015:99:2939-54.

4. Chundawat SP, Beckham GT, Himmel ME, Dale BE. Deconstruction of lignocellulosic biomass to fuels and chemicals. Annu Rev Chem Biomol Eng. 2011;2:121-45

5. Andlar M, Rezić T, Marđetko N, Kracher D, Ludwig R, Šantek B. Lignocellulose degradation: an overview of fungi and fungal enzymes involved in lignocellulose degradation. Eng Life Sci. 2018;18:768-78.

6. Himmel ME, Ding SY, Johnson DK, Adney WS, Nimlos MR, Brady JW, Foust TD. Biomass recalcitrance: engineering plants and enzymes for biofuels production. Science. 2007;315:804-7.

7. van Zyl WH, Lynd LR, den Haan R, McBride JE. Consolidated bioprocessing for bioethanol production using Saccharomyces cerevisiae. Adv Biochem Eng Biotechnol. 2007;108:205-35.

8. Olson DG, McBride JE, Shaw AJ, Lynd LR. Recent progress in consolidated bioprocessing. Curr Opin Biotechnol. 2012;23:396-405.

9. Fan Z. Chapter 7-consolidated bioprocessing for ethanol production. In: Qureshi N, Hodge DB, Vertès AA, editors. Biorefineries. Amsterdam: Elsevier; 2014. p. 141-60.

10. Yanase S, Yamada R, Kaneko S, Noda H, Hasunuma T, Tanaka T, Ogino C, Fukuda $\mathrm{H}$, Kondo A. Ethanol production from cellulosic materials using cellulase-expressing yeast. Biotechnol J. 2010;5:449-55.

11. Beopoulos A, Chardot T, Nicaud JM. Yarrowia lipolytica: a model and a tool to understand the mechanisms implicated in lipid accumulation. Biochimie. 2009;91:692-6.

12. Tsigie YA, Wang CY, Kasim NS, Diem QD, Huynh LH, Ho QP, Truong CT, Ju $\mathrm{YH}$. Oil production from Yarrowia lipolytica Po1g using rice bran hydrolysate. J Biomed Biotechnol. 2012:2012:378384

13. Lin J, Shen H, Tan H, Zhao X, Wu S, Hu C, Zhao ZK. Lipid production by Lipomyces starkeyi cells in glucose solution without auxiliary nutrients. J Biotechnol. 2011;152:184-8.

14. Sitepu IR, Jin M, Fernandez JE, da Costa SL, Balan V, Boundy-Mills KL. Identification of oleaginous yeast strains able to accumulate high intracellular lipids when cultivated in alkaline pretreated corn stover. Appl Microbiol Biotechnol. 2014:98:7645-57.

15. Zhao X, Kong X, Hua Y, Feng B, Zhao Z. Medium optimization for lipid production through co-fermentation of glucose and xylose by the oleaginous yeast Lipomyces starkeyi. Eur J Lipid Sci Technol. 2008;110:405-12. 
16. He Q, Yang Y, Yang S, Donohoe BS, Van Wychen S, Zhang M, Himmel ME, Knoshaug EP. Oleaginicity of the yeast strain Saccharomyces cerevisiae D5A. Biotechnol Biofuels. 2018;11:258.

17. Knoshaug EP, Wychen SV, Arjun S, Zhang M. Lipid accumulation from glucose and xylose in an engineered, naturally oleaginous strain of Saccharomyces cerevisiae. Biofuel Res J. 2018;5:800-5.

18. Wood TM. Fungal cellulases. Biochem Soc Trans. 1992;20:46-53.

19. Khatun MM, Yu X, Kondo A, Bai F, Zhao X. Improved ethanol production at high temperature by consolidated bioprocessing using Saccharomyces cerevisiae strain engineered with artificial zinc finger protein. Bioresour Technol. 2017;245:1447-54.

20. Hasunuma T, Kondo A. Development of yeast cell factories for consolidated bioprocessing of lignocellulose to bioethanol through cell surface engineering. Biotechnol Adv. 2012;30:1207-18.

21. Ilmen M, den Haan R, Brevnova E, McBride J, Wiswall E, Froehlich A, Koivula A, Voutilainen SP, Siika-Aho M, la Grange DC, et al. High level secretion of cellobiohydrolases by Saccharomyces cerevisiae. Biotechnol Biofuels. 2011:4:30.

22. Guo ZP, Duquesne S, Bozonnet S, Cioci G, Nicaud JM, Marty A, O'Donohue MJ. Conferring cellulose-degrading ability to Yarrowia lipolytica to facilitate a consolidated bioprocessing approach. Biotechnol Biofuels. 2017;10:132.

23. Guo Z-P, Duquesne S, Bozonnet S, Nicaud J-M, Marty A, O'Donohue MJ. Expressing accessory proteins in cellulolytic Yarrowia lipolytica to improve the conversion yield of recalcitrant cellulose. Biotechnol Biofuels. 2017;10:298.

24. Wei H, Wang W, Alahuhta M, Vander Wall T, Baker JO, Taylor LE, Decker SR, Himmel ME, Zhang M. Engineering towards a complete heterologous cellulase secretome in Yarrowia lipolytica reveals its potential for consolidated bioprocessing. Biotechnol Biofuels. 2014;7:148.

25. Xu Q, Knoshaug EP, Wang W, Alahuhta M, Baker JO, Yang S, Vander Wall T, Decker SR, Himmel ME, Zhang M, Wei H. Expression and secretion of fungal endoglucanase II and chimeric cellobiohydrolase I in the oleaginous yeast Lipomyces starkeyi. Microb Cell Fact. 2017;16:126.

26. Xu Q, Alahuhta M, Wei H, Knoshaug EP, Wang W, Baker JO, Vander Wall T, Himmel ME, Zhang M. Expression of an endoglucanase-cellobiohydrolase fusion protein in Saccharomyces cerevisiae, Yarrowia lipolytica, and Lipomyces starkeyi. Biotechnol Biofuels. 2018;11:322.

27. Boer H, Teeri TT, Koivula A. Characterization of Trichoderma reesei cellobiohydrolase Cel7A secreted from Pichia pastoris using two different promoters. Biotechnol Bioeng. 2000;69:486-94.

28. Lynd LR, van Zyl WH, McBride JE, Laser M. Consolidated bioprocessing of cellulosic biomass: an update. Curr Opin Biotechnol. 2005;16:577-83.

29. Kricka W, Fitzpatrick J, Bond U. Metabolic engineering of yeasts by heterologous enzyme production for degradation of cellulose and hemicelIulose from biomass: a perspective. Front Microbiol. 2014;5:174

30. Den Haan R, Kroukamp H, Van Zyl J-HD, Van Zyl WH. Cellobiohydrolase secretion by yeast: Current state and prospects for improvement. Process Biochem. 2013;48:1-12.

31. Lambertz C, Garvey M, Klinger J, Heesel D, Klose H, Fischer R, Commandeur $\mathrm{U}$. Challenges and advances in the heterologous expression of cellulolytic enzymes: a review. Biotechnol Biofuels. 2014;7:135.

32. Nakatani $Y$, Yamada R, Ogino C, Kondo A. Synergetic effect of yeast cell-surface expression of cellulase and expansin-like protein on direct ethanol production from cellulose. Microb Cell Fact. 2013;12:66.

33. Liu Z, Inokuma K, Ho SH, Haan R, Hasunuma T, van Zyl WH, Kondo A. Combined cell-surface display- and secretion-based strategies for production of cellulosic ethanol with Saccharomyces cerevisiae. Biotechnol Biofuels. 2015;8:162.

34. Ito Y, Yamanishi M, Ikeuchi A, Imamura C, Matsuyama T. Combinatorial screening for transgenic yeasts with high cellulase activities in combination with a tunable expression system. PLoS ONE. 2015;10:e0144870.

35. Lee CR, Sung BH, Lim KM, Kim MJ, Sohn MJ, Bae JH, Sohn JH. Co-fermentation using Recombinant Saccharomyces cerevisiae yeast strains hypersecreting different cellulases for the production of cellulosic bioethanol. Sci Rep. 2017;7:4428.

36. Takada G, Kawaguchi T, Sumitani J, Arai M. Expression of Aspergillus aculeatus No. F-50 cellobiohydrolase I (cbhl) and beta-glucosidase (bgl1) genes by Saccharomyces cerevisiae. Biosci Biotechnol Biochem. 1998;62:1615-8.

37. Penttila ME, Andre L, Lehtovaara P, Bailey M, Teeri TT, Knowles JK. Efficient secretion of two fungal cellobiohydrolases by Saccharomyces cerevisiae. Gene. 1988:63:103-12.

38. du Plessis L, Rose SH, van ZylWH. Exploring improved endoglucanase expression in Saccharomyces cerevisiae strains. Appl Microbiol Biotechnol. 2010;86:1503-11.

39. Den Haan R, McBride JE, Grange DCL, Lynd LR, Van Zyl WH. Functional expression of cellobiohydrolases in Saccharomyces cerevisiae towards one-step conversion of cellulose to ethanol. Enzyme and Microbial Technology. 2007;40:1291-9.

40. Godbole S, Decker SR, Nieves RA, Adney WS, Vinzant TB, Baker JO, Thomas SR, Himmel ME. Cloning and expression of Trichoderma reesei cellobiohydrolase I in Pichia pastoris. BiotechnolProg. 1999;15:828-33.

41. Xu Q, Gao W, Ding SY, Kenig R, Shoham Y, Bayer EA, Lamed R. The cellulosome system of Acetivibrio cellulolyticus includes a novel type of adaptor protein and a cell surface anchoring protein. J Bacteriol. 2003;185:4548-57.

42. Wang W, Wei H, Knoshaug E, Wychen S, Xu Q, Himmel ME, Zhang M. Fatty alcohol production in Lipomyces starkeyi and Yarrowia lipolytica. Biotechnol Biofuels. 2016;9:227.

43. Xu Q, Alahuhta M, Wei H, Knoshaug EP, Wang W, Baker JO, Vander Wall T, Himmel ME, Zhang M. Expression of an endoglucanase-cellobiohydrolase fusion protein in Saccharomyces cerevisiae, Yarrowia lipolytica, and Lipomyces starkeyi. Biotechnol Biofuels. 2018;11:322.

44. Vantilbeurgh $\mathrm{H}$, Tomme P, Claeyssens M, Bhikhabhai R, Pettersson G Limited Proteolysis of the cellobiohydrolase I from Trichoderma-Reeseiseparation of functional domains. FEBS Lett. 1986;204:223-7.

45. Schneider CA, Rasband WS, Eliceiri KW. NIH image to imageJ: 25 years of image analysis. Nat Methods. 2012;9:671-5.

46. Linger JG, Taylor LE 2nd, Baker JO, Vander Wall T, Hobdey SE, Podkaminer K, Himmel ME, Decker SR. A constitutive expression system for glycosyl hydrolase family 7 cellobiohydrolases in Hypocrea jecorina. Biotechnol Biofuels. 2015;8:45.

47. Schell DJ, Farmer J, Newman M, McMillan JD. Dilute-sulfuric acid pretreatment of corn stover in pilot-scale reactor: investigation of yields, kinetics, and enzymatic digestibilities of solids. Appl Biochem Biotechnol. 2003;105-108:69-85.

48. Baker JO, McCarley JR, Lovett R, Yu CH, Adney WS, Rignall TR, Vinzant TB, Decker SR, Sakon J, Himmel ME. Catalytically enhanced endocellulase Cel5A from Acidothermus cellulolyticus. Appl Biochem Biotechnol. 2005;121-124:129-48.

49. Reinikainen T, Ruohonen $L$, Nevanen T, Laaksonen L, Kraulis P, Jones TA, Knowles JK, Teeri TT. Investigation of the function of mutated cellulosebinding domains of Trichoderma reesei cellobiohydrolase I. Proteins. 1992;14:475-82.

50. Kukuruzinska MA, Bergh MLE, Jackson BJ. Protein glycosylation in yeast. Annu Rev Biochem. 1987;56:915-44.

51. Amore A, Knott BC, Supekar NT, Shajahan A, Azadi P, Zhao P, Wells L, Linger JG, Hobdey SE, Vander Wall TA, et al. Distinct roles of $\mathrm{N}$ - and O-glycans in cellulase activity and stability. Proc Natl Acad Sci U S A. 2017:114:13667-72.

52. Taylor LE 2nd, Knott BC, Baker JO, Alahuhta PM, Hobdey SE, Linger JG, Lunin W, Amore A, Subramanian V, Podkaminer K, et al. Engineering enhanced cellobiohydrolase activity. Nat Commun. 2018;9:1186.

53. Sola RJ, Griebenow K. Glycosylation of therapeutic proteins: an effective strategy to optimize efficacy. BioDrugs. 2010;24:9-21.

54. Guan X, Chaffey PK, Zeng C, Greene ER, Chen L, Drake MR, Chen C, Groobman A, Resch MG, Himmel ME, et al. Molecular-scale features that govern the effects of O-glycosylation on a carbohydrate-binding module. Chem Sci. 2015:6:7185-9.

\section{Publisher's Note}

Springer Nature remains neutral with regard to jurisdictional claims in published maps and institutional affiliations. 